\title{
ensembleTax: An R package for determinations of ensemble taxonomic assignments of phylogenetically-informative marker gene sequences
}

\author{
Dylan Catlett ${ }^{\text {Corresp., } 1}{ }^{,}$Kevin Son ${ }^{1}$, Connie Liang ${ }^{1}$ \\ ${ }^{1}$ Earth Research Institute, University of California, Santa Barbara, Santa Barbara, CA, United States \\ Corresponding Author: Dylan Catlett \\ Email address: dsc@ucsb.edu
}

Background. High-throughput sequencing of phylogenetically informative marker genes is a widely used method to assess the diversity and composition of microbial communities. Taxonomic assignment of sampled marker gene sequences (referred to as amplicon sequence variants, or ASVs) imparts ecological significance to these genetic data. To assign taxonomy to an ASV, a taxonomic assignment algorithm compares the ASV to a collection of reference sequences (a reference database) with known taxonomic affiliations. However, many taxonomic assignment algorithms and reference databases are available, and the optimal algorithm and database for a particular scientific question is often unclear. Here, we present the ensembleTax $\mathrm{R}$ package, which provides an efficient framework for integrating taxonomic assignments predicted with any number of taxonomic assignment algorithms and reference databases to determine ensemble taxonomic assignments for ASVs.

Methods. The ensembleTax R package relies on two core algorithms: taxmapper and assign.ensembleTax. The taxmapper algorithm maps taxonomic assignments derived from one reference database onto the taxonomic nomenclature (a set of taxonomic naming and ranking conventions) of another reference database. The assign.ensembleTax algorithm computes ensemble taxonomic assignments for each ASV in a data set based on any number of taxonomic assignments determined with independent methods. Various parameters allow analysts to prioritize obtaining either more ASVs with more predicted clade names or more robust clade name predictions supported by multiple independent methods in ensemble taxonomic assignments.

Results. The ensembleTax $\mathrm{R}$ package is used to compute two sets of ensemble taxonomic assignments for a collection of protistan ASVs sampled from the coastal ocean. Comparisons of taxonomic assignments predicted by individual methods with those predicted by ensemble methods show that conservative implementations of the ensembleTax package minimize disagreements between taxonomic assignments predicted by individual and ensemble methods, but result in ASVs with fewer ranks assigned taxonomy. Less conservative implementations of the ensembleTax package result in an increased fraction of ASVs classified at all taxonomic ranks, but increase the number of ASVs for which ensemble assignments disagree with those predicted by individual methods.

Discussion. We discuss how implementation of the ensembleTax R package may be optimized to address specific scientific objectives based on the results of the application of the ensembleTax package to marine protist communities. While further work is required to evaluate the accuracy of ensemble taxonomic assignments relative to taxonomic assignments predicted by individual methods, we also discuss scenarios where ensemble methods are expected to improve the accuracy of taxonomy prediction for ASVs.

PeerJ reviewing PDF | (2021:03:59589:1:0:NEW 3 Jun 2021) 
1

2 ensembleTax: An R package for determinations of

3 ensemble taxonomic assignments of phylogenetically-

4 informative marker gene sequences

5

6

7

8

9

10

11

12

13

14

15

16

17

18

19

20

21

22

23

24

25

26

27

28

29

30

31

32

33

34

35
Dylan Catlett ${ }^{1}$, Kevin Son ${ }^{1}$, Connie Liang ${ }^{1}$

${ }^{1}$ Earth Research Institute, UC Santa Barbara, Santa Barbara, CA, USA

Corresponding Author:

Dylan Catlett ${ }^{1}$

Ellison Hall rm. 5839, UC Santa Barbara, Santa Barbara, CA, 93106, USA

Email address: dsc@ucsb.edu 


\section{Abstract}

\section{Background.}

38 High-throughput sequencing of phylogenetically informative marker genes is a widely used method to assess the diversity and composition of microbial communities. Taxonomic assignment of sampled marker gene sequences (referred to as amplicon sequence variants, or ASVs) imparts ecological significance to these genetic data. To assign taxonomy to an ASV, a taxonomic assignment algorithm compares the ASV to a collection of reference sequences (a reference database) with known taxonomic affiliations. However, many taxonomic assignment algorithms and reference databases are available, and the optimal algorithm and database for a particular scientific question is often unclear. Here, we present the ensembleTax R package, which provides an efficient framework for integrating taxonomic assignments predicted with any number of taxonomic assignment algorithms and reference databases to determine ensemble taxonomic assignments for ASVs.

Methods.

The ensembleTax R package relies on two core algorithms: taxmapper and assign.ensembleTax. The taxmapper algorithm maps taxonomic assignments derived from one reference database onto the taxonomic nomenclature (a set of taxonomic naming and ranking conventions) of another reference database. The assign.ensembleTax algorithm computes ensemble taxonomic assignments for each ASV in a data set based on any number of taxonomic assignments determined with independent methods. Various parameters allow analysts to prioritize obtaining either more ASVs with more predicted clade names or more robust clade name predictions supported by multiple independent methods in ensemble taxonomic assignments.

\section{Results.}

The ensembleTax R package is used to compute two sets of ensemble taxonomic assignments for a collection of protistan ASVs sampled from the coastal ocean. Comparisons of taxonomic assignments predicted by individual methods with those predicted by ensemble methods show that conservative implementations of the ensembleTax package minimize disagreements between taxonomic assignments predicted by individual and ensemble methods, but result in ASVs with fewer ranks assigned taxonomy. Less conservative implementations of the ensembleTax package result in an increased fraction of ASVs classified at all taxonomic ranks, but increase the number of ASVs for which ensemble assignments disagree with those predicted by individual methods.

\section{Discussion.}

We discuss how implementation of the ensembleTax R package may be optimized to address specific scientific objectives based on the results of the application of the ensembleTax package to marine protist communities. While further work is required to evaluate the accuracy of ensemble taxonomic assignments relative to taxonomic assignments predicted by individual methods, we also discuss scenarios where ensemble methods are expected to improve the accuracy of taxonomy prediction for ASVs. 


\section{Introduction}

77

78

79

80

81

82

83

84

85

86

87

88

89

90

91

92

93

94

95

96

97

98

99

100

101

102

103

104

105

106

107

108

109

110

111

112

113

114

High-throughput amplicon sequencing of phylogenetically informative marker genes (also known as DNA meta-barcoding) is a widely used method for assessing the composition and diversity of microbial communities (Sogin et al., 2006; de Vargas et al., 2015). Commonly used phylogenetic marker genes include the $18 \mathrm{~S}$ small subunit ribosomal RNA gene (18S rDNA) in microbial eukaryotes, the 16S small subunit ribosomal RNA gene (16S rDNA) in prokaryotes, and the internal transcribed spacer region (ITS) in fungi (Woese and Fox, 1977; Medlin et al., 1988; Sogin et al., 2006; de Vargas et al., 2015). Marker gene sequencing studies typically rely on operational taxonomic units or amplicon sequence variants (ASVs) to serve as representatives of individual microbial species within a community (Schloss et al., 2011; Callahan et al., 2016; hereafter we use "ASV" to denote a marker gene sequence sampled from any system). Because an organism's taxonomy is often correlated with its ecology, assigning taxonomic identities to ASV sequences imparts ecological significance to genetic data. Taxonomic assignment thus represents a critical component of all marker gene sequencing studies.

Taxonomic assignment of marker gene sequences requires a reference database of marker gene sequences with known taxonomic identities, and an assignment algorithm that determines the most likely taxonomic affiliation of each representative ASV in a data set by comparing it to the sequences in a reference database. Reference databases are typically tailored to a specific group of organisms and/or a single marker gene (Guillou et al., 2013; Quast et al., 2013; Cole et al., 2014; Glöckner et al., 2017), although some include multiple marker genes from multiple groups of organisms (e.g., the SILVA reference database; Quast et al., 2013; Glöckner et al., 2017). However, different reference databases frequently employ disparate taxonomic naming and ranking conventions, and certain reference databases include subsets of reference sequences that are not found in other databases. For example, at the time of writing both the Protistan Ribosomal Reference database (pr2; Guillou et al., 2013) and the SILVA reference database (silva; Quast et al., 2013; Glöckner et al., 2017) include large collections of 18S rDNA reference sequences. The disparate naming and ranking conventions employed by silva and pr2 make it difficult for analysts of $18 \mathrm{~S}$ rDNA data sets to reconcile taxonomic assignments predicted using one database with those predicted using the other.

Analysts of marker gene sequence data sets must also choose from one of many taxonomic assignment algorithms, each of which employs unique methods and is associated with error (e.g. Wang et al., 2007; Bokulich et al., 2018; Edgar, 2018a; Murali et al., 2018). There are some assignment algorithms that consistently outperform others across multiple benchmark exercises, such as the RDP naïve Bayesian classifier (Wang et al., 2007), the recently introduced idtaxa algorithm (Murali et al., 2018), and others. However, realistic validation of taxonomic assignment algorithms is not straightforward (see Edgar, 2018a for a summary of approaches

Peerj reviewing PDF | (2021:03:59589:1:0:NEW 3 Jun 2021) 
115 used in benchmarking assignment algorithms). Thus, the optimal taxonomic assignment

116 algorithm and reference database for a particular scientific question or data set is often uncertain.

117

118 Ensemble approaches that integrate results from multiple independent bioinformatic methods

119 have been shown to improve the accuracy of assigning taxonomy to meta-genomic fragments,

120 and of assigning meta-genomic fragments to genomes (McIntyre et al., 2017; Sieber et al., 2018).

121 Similarly, ensemble approaches that incorporate taxonomic assignments from multiple

122 taxonomic assignment algorithms and/or reference databases may be expected to improve

123 taxonomic assignments of ASV sequences. To our knowledge, only one ensemble (or

124 "consensus") approach has been proposed for taxonomic assignment of fungal ITS sequences

125 (Gdanetz et al., 2017), but this method is not generalizable to prokaryotic 16S rDNA or

126 eukaryotic 18S rDNA sequences and does not enable the use of multiple reference databases

127 with disparate naming and ranking conventions.

128

129

Here we introduce the ensembleTax R package, which enables analysts of marker gene sequence

130 data to efficiently and flexibly compute ensemble (or "consensus") taxonomic assignments for each representative ASV sequence in a marker gene data set. Two core algorithms, taxmapper and assign.ensembleTax, allow users to map taxonomic assignments from one reference database onto another reference database's taxonomic nomenclature (see Table 1 for a glossary of terms used here), and to compute ensemble taxonomic assignments, respectively. The ensembleTax R package includes taxmapper, assign.ensembleTax, and additional pre-processing functions that enable streamlined ensemble taxonomic assignment determinations immediately following determination and initial taxonomic assignment of ASVs with the dada2 and DECIPHER $\mathrm{R}$ packages (Callahan et al., 2016; Wright, 2016; Murali et al., 2018). We demonstrate the utility and flexibility of the ensembleTax R package using a collection of protistan ASVs derived from the V9 hypervariable region of the $18 \mathrm{~S}$ rDNA sampled from the coastal ocean. In particular, we demonstrate how the ensembleTax R package allows investigators to prioritize obtaining either more predicted clade names for more ASVs or more robust taxonomic assignment predictions supported by multiple independent methods in ensemble taxonomic assignments. The ensembleTax R package is freely available on Github (https://github.com/dcat4/ensembleTax/blob/master/README.md), and CRAN (https://cran.rstudio.com/web/packages/ensembleTax/index.html), and will continue to be developed as taxonomic assignment methods and reference databases continue to evolve.

ensembleTax package overview

152

\section{Package Description and Implementation} and built with $\mathrm{R}$ version 3.6.2 (R Core Team, 2019). The R package devtools (Wickham et al., 
155

156

157

158

159

160

161

162

163

164

165

166

167

168

169

170

171

172

173

174

175

176

177

178

179

180

181

182

183

184

185

186

187

188

189

190

191

192

193

2020) was used extensively to build the ensembleTax package. The ensembleTax package relies on other R packages including dplyr (Wickham et al., 2020), stringr (Wickham et al., 2019), DECIPHER (Wright, 2016), Biostrings (Pagès et al., 2019), and usethis (Wickham and Bryan, 2020). Vignettes were built with the knitr (Xie, 2020) and rmarkdown (Allaire et al., 2020) packages. The ensembleTax package is freely available for use under an MIT license, and can be installed from Github or CRAN (see https://github.com/dcat4/ensembleTax/blob/master/README.md for download instructions).

The ensembleTax R package was developed to offer an automated, efficient, and flexible tool for microbial ecologists to synthesize taxonomic assignment predictions made with any number of taxonomic assignment algorithms and/or reference databases. Table 1 provides a glossary of terms used here and within the documentation of the ensembleTax package, and Figure 1 outlines possible ensembleTax workflows for different taxonomic assignment methods. All ensembleTax workflows consist of converting the outputs of taxonomic assignment algorithms into dataframes that include ASV-identifying meta-data and corresponding taxonomic assignments for an arbitrary number of ranks, mapping of taxonomic assignments onto a common taxonomic nomenclature (if necessary), and determination of ensemble taxonomic assignments (Fig. 1). The ensembleTax package was developed to offer convenient analysis following ASV determination and initial taxonomic assignment with the dada2 and DECIPHER R packages (Callahan et al., 2016; Wright, 2016; Murali et al., 2018), but other methods for ASV determination and initial taxonomic assignment may be used prior to the ensembleTax workflow (Fig. 1).

The taxonomic assignment algorithms explicitly supported in the ensembleTax package are the Ribosomal Database Project (RDP) naïve Bayesian classifier (Wang et al., 2007) implemented in the dada2 R package (Callahan et al., 2016), and the recently developed idtaxa algorithm (Murali et al., 2018) implemented in the DECIPHER R package (Wright, 2016). Explicitly supported reference databases include the SILVA SSU non-redundant reference database v138 (Quast et al., 2013; Glöckner et al., 2017; henceforth, silva), the Protistan Ribosomal Reference database v4.12.0 (Guillou et al., 2013; henceforth, pr2), the GreenGenes reference database v13.8 clustered at 97\% similarity (DeSantis et al., 2006; McDonald et al., 2012), and the RDP 16S rRNA train set v16 (Cole et al., 2014). Additional assignment algorithms and/or reference databases can be incorporated so long as the data can be read into $\mathrm{R}$ and converted to a dataframe object. We welcome external contributions of $\mathrm{R}$ implementations of other taxonomic assignment algorithms and/or reference database data, and will continue to accommodate new methods as they are developed.

\section{Description of core algorithms and package data}


194 As noted above, taxmapper and assign.ensembleTax are the core algorithms for computing

195

196

197

198

199

200

201

202

203

204

205

206

207

208

209

210

211

212

213

214

215

216

217

218

219

220

221

222

223

224

225

226

227

228

229

230

231

232 ensemble taxonomic assignments with the ensembleTax package. taxmapper maps, or "translates", the taxonomic nomenclature used by one reference database onto the taxonomic nomenclature used by another, while assign.ensembleTax computes ensemble taxonomic assignments from taxonomic assignments generated by any number of unique taxonomic assignment algorithm and reference database combinations. Here we describe these algorithms and parameters that may be altered to tailor their performance for particular scientific objectives.

taxmapper maps the taxonomic nomenclature of one reference database onto the taxonomic nomenclature of another via rank-agnostic exact name matching. Figure 2 provides an example to demonstrate the mapping procedure used by taxmapper for several different variations in userspecified parameters. For each ASV in a data set, the taxonomic name at the lowest annotated rank (e.g. species if annotated, otherwise genus, etc.) is compared to all taxonomic names at the lowest annotated rank of the target taxonomic nomenclature. If an exact match is found in the reference taxonomic nomenclature onto which assignments are being mapped, the ASV is assigned the matched taxonomic name along with all higher taxonomic names according to the reference nomenclature, is not assigned (assigned NA) at all lower ranks, and mapping is complete for the ASV (Fig. 2). If an exact match is not found, the name is searched at higher ranks within the target taxonomic nomenclature. If an exact match is still not found, depending on user-controlled inputs (see below and Fig. 2) taxmapper either: collects taxonomic synonyms for the name being mapped, reformats the name and its taxonomic synonyms, and searches for each possible alternative name until a match is found; or, repeats the search for the taxonomic name assigned to the ASV at the next lowest annotated rank (e.g. genus if the species assignment was not successfully mapped). taxmapper returns the input ASV-identifying data with taxonomic assignments mapped onto the specified reference database's taxonomic nomenclature (Fig. 2). If specified by the user, taxmapper also returns the taxonomic names for which no exact match was found in the reference taxonomic nomenclature, as well as the "mapping rubric" containing all unique ASV taxonomic assignments supplied by the user and their corresponding mapped taxonomic assignments using the reference nomenclature.

The approach taken by taxmapper for mapping taxonomic assignments implicitly assumes that a taxonomic name has equivalent meaning regardless of the reference database in which the taxonomic name is found. This assumption is violated in reference nomenclatures that employ ambiguous terms as standalone taxonomic names such as "incertae sedis", "Clade_X", "Group_2", etc. (a complete list is provided in the package documentation). In the event such taxonomic names are assigned to an ASV and encountered by taxmapper, the taxonomic name at the lowest unambiguously annotated rank is appended to the ambiguous name. Exact name matching then proceeds as described above for the newly created, unambiguous taxonomic name (Fig. 2).

Peer) reviewing PDF | (2021:03:59589:1:0:NEW 3 Jun 2021) 
233 Several optional arguments may be supplied to taxmapper to increase the number of taxonomic 234 names mapped (Fig. 2). Users may relax the exact name-matching employed by taxmapper with 235 the ignore.format argument. This relaxation attempts to account for common formatting 236 differences between reference databases (such as the interchangeable use of underscores, 237 hyphens, or spaces, as in Pseudonitzchia vs. Pseudo-nitzchia in Fig. 2; a complete list of 238 formatting variants for which exact matches are searched is available in the package 239 documentation).

240

241 Users may also consider taxonomic synonyms supplied with the ensembleTax package, or 242 provide taxmapper with a custom synonym file that includes a collection of taxonomic names 243 and known synonyms. Synonyms are searched in the event that an exact match for a particular 244 taxonomic name is not found in the taxonomic nomenclature onto which the name is being 245 mapped (Fig. 2). The ensembleTax package includes a compilation of eukaryotic taxonomic 246 synonyms that were compiled manually based on the NCBI Taxonomy Browser (Benson et al., 247 2012), the World Register of Marine Species (WoRMS Editorial Board, 2020), Wikispecies 248 (https://species.wikimedia.org/wiki/Main_Page), the Integrated Taxonomic Information System 249 (http://www.itis.gov), the Tree of Life Web Project (Maddison and Schulz, 2007), and various 250 other literature sources (Silén, 1972; Cavalier-Smith, 1993; Adl et al., 2005; Casu and CuriniGalletti, 2006; Gomez et al., 2010; Ratnasingham and Hebert, 2007; Adl et al., 2012; Braun, 2018; Hibbett et al., 2018; Varol et al., 2018; Adl et al., 2019). AlgaeBase (Guiry and Guiry, 2020) was used to identify some sources of primary literature containing taxonomic synonyms cited above. Users may also supply custom collections of taxonomic synonyms for use with taxmapper.

If taxonomic names at the basal rank should be retained in the mapped taxonomy table regardless of whether they are found in the taxonomic nomenclature onto which they are being mapped, these may be supplied to taxmapper as exceptions. These names will be assigned to the basal rank of the mapped taxonomy table (kingdom or domain in most reference database taxonomic nomenclatures), with all other ranks unassigned. This option is intended for cases where assignments of non-target organisms are important. For example, if a universal primer set is used to amplify a marker gene from both eukaryotes and prokaryotes (e.g. the $16 \mathrm{~S}$ and $18 \mathrm{~S}$ rDNA as in Parada et al., 2016), it may be necessary to retain prokaryote assignments when mapping onto a eukaryote-specific taxonomic nomenclature, or vice-versa.

The assign.ensembleTax algorithm computes ensemble taxonomic assignments based on any number of input taxonomy tables that share a common taxonomic nomenclature. Figure 3 illustrates several example ensemble determinations based on two different examples of input taxonomic assignments and considering adjustments to several assign.ensembleTax parameters. Ensemble assignments are computed independently for each ASV and each taxonomic rank by finding the highest-frequency taxonomic assignment across all input taxonomy tables. If 
273 particular taxonomy tables are likely to include more robust taxonomic assignments, the user 274 may weight taxonomic assignments in these tables more highly than others using the weights 275 argument. In the event that two or more taxonomic assignments are found at equal weighted 276 (based on the weights argument) maximum frequencies, the ensemble taxonomic assignment is 277 unassigned (assigned NA; Fig. 3). This behavior can be modified by specifying the names of 278 taxonomy tables whose assignments should be prioritized in the event that two or more 279 taxonomic assignments are found at equal weighted maximum frequencies (the tiebreakz 280 argument; Fig. 3A). By default, non-assignments (represented by NA) are considered when

281

282

283

284

285

286

287

288

289

290

291

292

293

294

295

296

297

298

299

300

301

302

303

304

305

306

307

308

309

310

311

312 determining ensemble taxonomic assignments, and the ensemble is assigned NA when this is the highest-frequency assignment (Fig. 3). Non-assignments can be excluded from ensemble taxonomic assignment calculations using the count.na argument (Fig. 3B). Finally, users may specify the minimum proportion of input taxonomy tables that a taxonomic assignment must be found in in order to be assigned as the ensemble taxonomic assignment using the assign.threshold argument (Fig. 3B).

\section{Application of ensembleTax to natural marine protist communities}

\section{Data set overview and application of the ensembleTax package}

To demonstrate that the ensembleTax package can be used to compute ensemble taxonomic assignments optimized for a variety of scientific objectives, we used the ensembleTax R package to determine ensemble taxonomic assignments for 15447 protistan ASVs inferred from 358 samples of marine plankton communities from the Santa Barbara Channel, CA. The ASVs are derived from the V9 hypervariable region of the 18S rDNA. Wet lab methods are described in Catlett et al. (2020). ASVs were determined using dada2 (Callahah et al., 2016) following Catlett et al. (2020), and four independent taxonomic assignments were determined for each ASV by implementing the RDP naïve Bayesian classifier (Wang et al., 2007) with a bootstrap confidence threshold of $60 \%$ and the idtaxa algorithm (Murali et al., 2018) with a bootstrap confidence threshold of $50 \%$ against both the $\mathrm{pr} 2$ and silva reference databases. These four sets of taxonomic assignments are referred to as bayes-pr2, bayes-silva, idtax-pr2, and idtax-silva. Assignments generated using the silva database were mapped onto the $\mathrm{pr} 2$ taxonomic nomenclature, and assignments generated with the $\mathrm{pr} 2$ database were mapped onto the silva taxonomic nomenclature using taxmapper and considering the taxonomic synonyms included with the ensembleTax package, as well as formatting variants of these names (ignore.format $=$ TRUE, synonym.file = "default"). Bacteria and Archaea assignments were maintained when mapping bayes-silva and idtax-silva onto pr2 since pr2 does not include these domains/kingdoms (exceptions $=c($ "Archaea", "Bacteria")).

In order to illustrate that assign.ensembleTax parameters can be adjusted to balance trade-offs between obtaining more ASVs with assigned taxonomy or more robust taxonomic assignments 
313 supported by multiple methods, two different collections of ensemble taxonomic assignments

314 were computed from the four independent taxonomy tables generated with each reference

315 databases' taxonomic nomenclature. The first favored ensemble taxonomic assignments

316 supported by multiple individual methods (ensemble1-pr2 and ensemble1-silva), and the second

317 favored obtaining more predicted clade names for a larger proportion of ASVs (ensemble2-pr2

318 and ensemble2-silva). Annotations supported by multiple independent methods were prioritized

319 in ensemble1-pr2 and ensemble1-silva by including non-assignments in ensemble determinations

320 (count.na $=T R U E$ ), and by specifying that no single taxonomy table should be favored in the

321

322

323

324

325

326

327

328

329

330

331

332

333

334

335

336

337

338

339

340

341

342

343

344

345

346

347

348

349

350

351

352 event that different taxonomic assignments were found at equivalent maximum frequencies across the input taxonomy tables (tiebreakz $=N U L L)$. An increased proportion of ASVs with more predicted clade names was prioritized in ensemble2-pr2 and ensemble2-silva by ignoring non-assignments (count.na $=F A L S E$ ), and by prioritizing assignments from specific taxonomy tables when multiple taxonomic assignments were found at equivalent maximum frequencies across the input taxonomy tables. In such events, assignments found in idtax-pr2, idtax-silva, and bayes-pr2, respectively, were prioritized in ensemble2-pr2 (tiebreakz $=c$ ("idtax-pr2", "idtaxsilva", "bayes-pr2")), while idtax-silva, idtax-pr2, and bayes-silva, respectively, were prioritized in ensemble2-silva (tiebreakz $=c$ ("idtax-silva", "idtax-pr2", "bayes-silva")). In order to focus our analysis on protists, ASVs assigned to Archaea, Bacteria, Metazoa, Fungi, or Streptophyta according to ensemble2-pr2, as well as ASVs that were $<90$ or $>180 \mathrm{nt}$ (target amplicon is 120$130 \mathrm{nt}$ ) in length, were discarded prior to further analysis.

\section{Results}

We compared the taxonomic assignments obtained with each of the four individual methods (bayes-pr2, bayes-silva, idtax-pr2, idtax-silva) to the two collections of ensemble taxonomic assignments (ensemble1 and ensemble2) using both the pr2 (Fig. 2A, 2C, 2E) and silva (Fig. 2B, $2 \mathrm{D}, 2 \mathrm{~F})$ taxonomic nomenclatures. To investigate differences in the number of predicted clade names between the ensemble and individual taxonomic assignment methods at different levels of the taxonomic hierarchy, we performed rank-wise comparisons of the proportion of ASVs that remained unassigned in each taxonomy table (Fig. 2A-B). We also calculated the proportion of ASVs for which taxonomic assignments predicted with one individual or ensemble method were assigned to more or less ranks, perfectly agreed, or disagreed (at any rank) with each of the two collections of ensemble taxonomic assignments (Fig. 2C-F). The latter comparisons were performed to assess the rates of agreement and disagreement between individual and ensemble methods using different assign.ensembleTax parameters.

Comparisons of the proportion of unassigned ASVs at each taxonomic rank across the six taxonomy tables demonstrate that substantially more taxonomic names can be assigned to ASVs at all taxonomic ranks with certain implementations of the ensembleTax package (Fig. 2A and 2B). The ensemble 2 taxonomy table had the lowest proportion of ASVs unassigned at all ranks 
353

354

355

356

357

358

359

360

361

362

363

364

365

366

367

368

369

370

371

372

373

374

375

376

377

378

379

380

381

382

383

384

385

386

387

388

389

390

391

392

across both the silva and pr2 taxonomic nomenclatures, with the largest increase in the number of taxonomic names assigned at higher ranks (kingdom/domain to class). Notably, $>13 \%$ more ASVs were assigned to a class in the ensemble2 taxonomy table relative to any other taxonomy table using both taxonomic nomenclatures. With the silva taxonomic nomenclature, nearly $20 \%$ more ASVs were assigned to a class in the ensemble2 taxonomy table than with any other method. Conversely, in the ensemble1 taxonomy table the proportion of ASVs that remained unassigned was typically higher than all but one or two of the other taxonomy tables. However, where taxonomic names are assigned in the ensemblel taxonomy table, they are expected to be robust as they were predicted by two or more of the independent taxonomic assignment methods.

Comparisons of the taxonomic assignments predicted for each ASV by each independent taxonomic assignment method with the two collections of ensemble taxonomic assignments demonstrate that the parameter space of the assign.ensembleTax algorithm can be optimized to address different scientific objectives (Fig. 2C-F). In comparisons with the ensemble1 taxonomy table, a higher proportion of ASVs were assigned taxonomy at more ranks with individual taxonomic assignment methods $(9.3-49.1 \%$ with the pr2 nomenclature; $22.2-32.8 \%$ with the silva nomenclature) than were assigned at less ranks with individual methods $(6.3-27.4 \%$ with the pr2 nomenclature; $3.1-19.5 \%$ with the silva nomenclature). This result reflects the conservative approach used to compute ensemble assignments in the ensemble1 taxonomy table. Conversely, relative to the ensemble 2 taxonomy table, $30 \%$ or more of the ASVs were assigned taxonomy at less ranks by all independent taxonomic assignment methods, again demonstrating the substantial increase in the number of ASVs that can be assigned taxonomy at lower ranks with less conservative implementations of the ensembleTax package.

Generally, where taxonomic names were assigned, rates of disagreement were low $(<10 \%)$ for all independent taxonomic assignment methods when compared with either set of ensemble assignments. Rates of disagreement were lower in comparisons with the ensemble1 taxonomy table $(0.7-2.2 \%$ with the pr 2 nomenclature; $<2 \%$ with the silva nomenclature except for $6.6 \%$ for idtax-silva) than in comparisons with the ensemble 2 taxonomy table $(2.2-5.8 \%$ with the $\mathrm{pr} 2$ nomenclature; $3.9-8.4 \%$ with the silva nomenclature). This result suggests that predicting more clade names for more ASVs with less conservative assign.ensembleTax implementations comes at the cost of greater uncertainty in taxonomy predictions and a likely increase in false positive assignments. Interestingly, no conflicting taxonomic assignments were found when comparing the two ensemble taxonomy tables with one another for either taxonomic nomenclature, meaning the two assign.ensembleTax parameter spaces resulted in identical taxonomic assignments where ASVs were assigned. Differences in the number of ASVs with taxonomy assigned at lower ranks were large however, with 67 and $70 \%$ of ASVs classified to a lower rank in the ensemble 2 taxonomy table for the silva and pr2 taxonomic nomenclatures, respectively.

\section{Discussion}

Peer] reviewing PDF | (2021:03:59589:1:0:NEW 3 Jun 2021) 
393

394

395

396

397

398

399

400

401

402

403

404

405

406

407

408

409

410

411

412

413

414

415

416

417

418

419

420

421

422

423

424

425

426

427

428

429

430

431

432

\section{Optimizing ensemble assignments for different scientific objectives}

The ensembleTax R package allows analysts of phylogenetically-informative marker gene sequence data to compute ensemble taxonomic assignments by integrating taxonomic assignments predicted with any number of independent methods (Figs. 1-3). The use of multiple reference databases employing disparate taxonomic nomenclatures is enabled by the taxmapper algorithm, which maps one taxonomic nomenclature onto another by rank-agnostic exact name matching (Fig. 2). The assign.ensembleTax algorithm computes ensemble taxonomic assignments and includes a suite of parameters that can be modified to optimize trade-offs between predicting more clade names for more ASVs or only assigning taxonomic names that are supported by multiple independent methods (Fig. 3).

Application of the ensembleTax package to determine ensemble taxonomic assignments for a large collection of protistan ASVs sampled from the coastal ocean confirmed that different parameter spaces can be implemented in the ensembleTax package to optimize ensemble assignments for different scientific objectives (Fig. 4). Conservative implementations of assign.ensembleTax can be achieved by counting non-assignments (count.na $=T R U E$ ), by leaving an ASV unassigned when conflicting assignments are found at equal maximum frequencies across the independent assignment methods considered (tiebreakz $=N U L L)$, and/or by increasing the proportion of independent methods that must predict a taxonomic assignment for it to be assigned to the ensemble (assign.threshold $=0.5$ or assign.threshold $=1$ ). Conservative ensemble determinations result in minimal disagreements in taxonomy predictions between the ensemble and independent methods indicating strong support for taxonomy predictions across methods (Fig. 4C-D), but also result in fewer ASVs with predicted taxonomic names in the ensemble (Fig. 4). These methods are thus well-suited to scientific applications that require more robust, well-supported taxonomy predictions and where obtaining taxonomy predictions for fewer ASVs can be tolerated, such as studies that rely heavily on taxonomic assignments at lower ranks and/or that focus on the diversity or ecology of a particular taxonomic group.

Conversely, less conservative ensemble assignments can be determined by removing nonassignments from consideration (count.na $=F A L S E$ ), by prioritizing certain methods in the event that conflicting assignments are found at the highest frequency (tiebreakz, weights), and/or by reducing the proportion of taxonomy tables that must corroborate a taxonomic assignment for it to be prescribed to the ensemble (assign.threshold $=0$ ). Such implementations increase the number of ASVs with assigned taxonomic names at all ranks, but also increase the number of ASVs with conflicting taxonomic assignments between individual methods and the ensemble (Fig. 4). Thus, less conservative implementations of the ensembleTax package are better suited for scientific applications where assigning taxonomy to a higher proportion of ASVs is needed

Peer) reviewing PDF | (2021:03:59589:1:0:NEW 3 Jun 2021) 
433 and where false positive annotations can be tolerated, such as studies focused on very broad 434 taxonomic groupings (supergroups, divisions, etc.). However, it should be noted that the increase 435 in ASVs with predicted taxonomy comes at the likely expense of an increase in incorrect 436 taxonomic assignments. Therefore, while taxonomic assignments of ASVs at lower ranks (e.g., 437 family, genus) should generally be interpreted with caution (Murali et al., 2018; Edgar, 2018a), 438 this is especially true for less conservative implementations of the ensembleTax package.

439 Overall, the ensembleTax package enables computation of optimized ensemble taxonomic

440

441

442

443

444

445

446

447

448

449

450

451

452

453

454

455

456

457

458

459

460

461

462

463

464

465

466

467

468

469

470

471

472 assignments for a wide variety of scientific questions.

\section{Potential for ensemble methods to increase the accuracy of taxonomy predictions}

Recent studies have highlighted errors and conflicts in taxonomic annotations of reference sequences included in widely used reference databases, as well as numerous difficulties associated with realistic validation of taxonomic assignment algorithms (Edgar, 2018a, 2018b; Murali et al., 2018). While the comparisons of taxonomic assignments of protistan ASVs predicted by individual and ensemble methods above demonstrate the versatility of ensemble methods (Fig. 4), we do not demonstrate nor imply that any individual or ensemble method provides more accurate taxonomy predictions than any other method. Future work should thus be devoted to robust validations of ensemble methods applied to particular marker genes. Nonetheless, in some cases ensemble methods may be expected to reduce the impacts of taxonomic assignment errors introduced by individual methods on downstream analyses of amplicon sequencing data. Here we discuss some example scenarios where ensemble methods may be expected to reduce the propagation of taxonomic assignment errors to downstream analyses.

Ensemble taxonomic assignments may be expected to reduce the impacts of errors in reference database annotations on downstream analyses of amplicon data. Sequence annotation errors are known to exist in large reference databases, and conflicts in the taxonomic annotation of a single reference sequence found in multiple databases have been documented (Edgar, 2018b).

Erroneous and/or conflicting annotations of a reference sequence should result in incorrect taxonomy predictions for any sampled ASV that is derived from or closely related to the incorrect reference sequence (Edgar, 2018b). Figure 3A shows that ensemble methods may mitigate the impacts of such errors on downstream analyses. To illustrate this point, assume the two conflicting taxonomic assignments shown in Figure $3 \mathrm{~A}$ arise due to conflicting annotations of the same reference sequence found in different reference databases (e.g., assignments by methods 1 and 2 were generated by implementing the same assignment algorithm with two different reference databases). A conservative (including the default) implementation of the assign.ensembleTax algorithm will not assign taxonomy where the individual methods disagree (Fig. 3A). Since at least one of these assignments must be incorrect, ensemble methods effectively remove the erroneous assignment from downstream analyses. If reference sequence 
473 annotation errors are less likely in one reference database than another, the assign.ensembleTax

474 parameter space can be modified to favor taxonomic assignments using the reference database

475 that is less error-prone (Fig. 3A). If a reference database is more error-prone for some taxonomic

476 groups and less error-prone for others, this strategy can be extended by computing ensemble

477 assignments separately for different subsets of ASVs. ASVs and their corresponding ensemble

478 taxonomic assignments can then be merged back into a single taxonomy table with a common

479 nomenclature with the use of taxmapper. Altogether, this example suggests the ensembleTax

480 package may improve taxonomic assignments by enabling streamlined integration of taxonomy

481 predictions based on multiple reference databases.

482

483

484

Ensemble methods may also be expected to reduce the impacts of taxonomic assignment errors

485 introduced by different assignment algorithms. Taxonomic assignment algorithms are known to introduce various types of error in taxonomic assignments of ASVs (Bokulich et al., 2018; Edgar, 2018a; Murali et al., 2018). Most widely used taxonomic assignment algorithms differ in the analytical approaches used to predict taxonomy, resulting in differences in observed error rates across a variety of validation exercises (Bokulich et al., 2018; Edgar, 2018a; Murali et al., 2018). Ignoring annotation errors in reference databases (see above), it is thus reasonable to assume that error profiles associated with different sequence classification approaches are independent from one another. Independent error profiles across different taxonomic assignment algorithms should lead to situations where a single assignment algorithm predicts an incorrect taxonomic assignment for an ASV that is not corroborated by other assignment algorithms (e.g., the assignment by method "m1" in Fig. 3B may be erroneous). In these situations, conservative implementations of the assign.ensembleTax algorithm can again reduce the impacts of taxonomic assignment errors on downstream analyses (Fig. 3B). Overall, the above conceptual examples demonstrate the potential for ensemble methods to improve accuracy in taxonomic assignments of marker gene sequence data, though further research is required to evaluate the accuracy of

500 ensemble methods relative to individual methods and to optimize ensemble methods for specific

501

\section{Conclusions}

503

504 We present the ensembleTax R package, including algorithms for flexible computations of 505 ensemble taxonomic assignments of phylogenetic marker gene sequence data. The two core 506 algorithms, taxmapper and assign.ensembleTax, compute ensemble taxonomic assignments from 507 any combination of taxonomic assignment algorithms and reference databases (Figs. 1-3). The 508 package data includes pre-compiled taxonomic nomenclatures from several widely used 509 reference databases, as well as a collection of eukaryotic taxonomic synonyms that can improve 510 the performance of taxmapper when used with eukaryotic ASVs. Use of the ensembleTax

511 package to compute ensemble taxonomic assignments on a eukaryotic 18S-V9 rDNA data set 512 from the coastal ocean showed that parameters can be tuned to optimize ensemble taxonomic 
513 assignments for specific scientific questions and objectives (Fig. 4). Further development of the 514 ensembleTax package will continue to expand the breadth of taxonomic assignment algorithms 515 and reference databases explicitly supported, and to ensure the most up-to-date versions of 516 reference databases remain easily accessible. Contributions from the community are welcome in 517 these areas. Given the potential of ensemble methods to improve taxonomic assignments of 518 marker gene sequences, future work should be devoted to robust evaluations of the performance 519 of ensemble taxonomic assignment methods relative to that of individual methods, and to 520 determining optimal ensemble inputs and parameters for particular groups of organisms, 521 phylogenetic marker genes, and scientific questions.

522

523

524

525

526

527

528

529

530

531

532

533

534

535

536

537

538

539

540

541

542

543

544

545

546

547

548

549

550

551

552

553

Adl, S.M., Simpson, A.G., Farmer, M.A., Andersen, R.A., Anderson, O.R., Barta, J.R., Bowser, S.S., Brugerolle, G.U.Y., Fensome, R.A., Fredericq, S. and James, T.Y., 2005. The new higher level classification of eukaryotes with emphasis on the taxonomy of protists. Journal of Eukaryotic Microbiology, 52(5), pp.399-451. https://doi.org/10.1111/j.1550-7408.2005.00053.x.

Adl, S.M., Simpson, A.G., Lane, C.E., Lukeš, J., Bass, D., Bowser, S.S., Brown, M.W., Burki, F., Dunthorn, M., Hampl, V. and Heiss, A., 2012. The revised classification of eukaryotes. Journal of Eukaryotic Microbiology, 59(5), pp.429-514. https://doi.org/10.1111/j.1550-7408.2012.00644.x.

Adl, S.M., Bass, D., Lane, C.E., Lukeš, J., Schoch, C.L., Smirnov, A., Agatha, S., Berney, C., Brown, M.W., Burki, F. and Cárdenas, P., 2019. Revisions to the classification, 
554

555

556

557

558

559

560

561

562

563

564

565

566

567

568

569

570

571

572

573

574

575

576

577

578

579

580

581

582

583

584

585

586

587

588

589

590

591

592

593

594

595

596

597

598

599

nomenclature, and diversity of eukaryotes. Journal of Eukaryotic Microbiology, 66(1), pp.4-119. https://doi.org/10.1111/jeu.12691.

JJ Allaire and Yihui Xie and Jonathan McPherson and Javier Luraschi and Kevin Ushey and Aron Atkins and Hadley Wickham and Joe Cheng and Winston Chang and Richard Iannone (2020). rmarkdown: Dynamic Documents for R. R package version 2.1. URL https://rmarkdown.rstudio.com.

Benson, D.A., Cavanaugh, M., Clark, K., Karsch-Mizrachi, I., Lipman, D.J., Ostell, J. and Sayers, E.W., 2012. GenBank. Nucleic acids research, 41(D1), pp.D36-D42. https://doi.org/10.1093/nar/gks1195.

Bokulich, N.A., Kaehler, B.D., Rideout, J.R., Dillon, M., Bolyen, E., Knight, R., Huttley, G.A., Caporaso, J.G., 2018. Optimizing taxonomic classification of marker-gene amplicon sequences with QIIME 2's q2-feature-classifier plugin. Microbiome 6, 90. https://doi.org/10.1186/s40168-018-0470-z.

Braun, U., 2018. Nomenclature of the fungal genus name Albigo. Schlechtendalia, 34, pp.91-94.

Callahan, B.J., McMurdie, P.J., Rosen, M.J., Han, A.W., Johnson, A.J.A. and Holmes, S.P., 2016. DADA2: high-resolution sample inference from Illumina amplicon data. Nature methods, 13(7), pp.581-583. https://doi.org/10.1038/nmeth.3869.

Casu, M. and Curini-Galletti, M., 2006. Genetic evidence for the existence of cryptic species in the mesopsammic flatworm Pseudomonocelis ophiocephala (Rhabditophora: Proseriata). Biological Journal of the Linnean Society, 87(4), pp.553-576. https://doi.org/10.1111/j.1095-8312.2006.00588.x.

Catlett, D., Matson, P.G., Carlson, C.A., Wilbanks, E.G., Siegel, D.A. and Iglesias-Rodriguez, M.D., 2020. Evaluation of accuracy and precision in an amplicon sequencing workflow for marine protist communities. Limnology and Oceanography: Methods, 18(1), pp.2040. https://doi.org/10.1002/lom3.10343.

Cavalier-Smith, T., 1993. Kingdom protozoa and its 18 phyla. Microbiology and Molecular Biology Reviews, 57(4), pp.953-994.

Cole, J. R., Q. Wang, J. A. Fish, B. Chai, D. M. McGarrell, Y. Sun, C. T. Brown, A. PorrasAlfaro, C. R. Kuske, and J. M. Tiedje. 2014. Ribosomal Database Project: data and tools for high throughput rRNA analysis. Nucleic Acids Research. 42(Database issue):D633D642. https://doi.org/10.1093/nar/gkt1244.

DeSantis, T.Z., Hugenholtz, P., Larsen, N., Rojas, M., Brodie, E.L., Keller, K., Huber, T., Dalevi, D., Hu, P. and Andersen, G.L., 2006. Greengenes, a chimera-checked 16S rRNA gene database and workbench compatible with ARB. Applied and environmental microbiology, 72(7), pp.5069-5072. https://doi.org/10.1128/AEM.03006-05. 
600 De Vargas, C., Audic, S., Henry, N., Decelle, J., Mahé, F., Logares, R., Lara, E., Berney, C., Le 601 Bescot, N., Probert, I. and Carmichael, M., 2015. Eukaryotic plankton diversity in the

602

603

604

605

606

607

608

609

610

611

612

613

614

615

616

617

618

619

620

621

622

623

624

625

626

627

628

629

630

631

632

633

634

635

636

637

638

639

640

641

642

643

644

645 sunlit ocean. Science, 348(6237). https://doi.org/10.1126/science.1261605.

Edgar, R.C., 2018a. Accuracy of taxonomy prediction for 16S rRNA and fungal ITS sequences. PeerJ, 6, p.e4652. https://doi.org/10.7717/peerj.4652.

Edgar R. 2018b. Taxonomy annotation and guide tree errors in 16S rRNA databases. PeerJ 6:e5030. https://doi.org/10.7717/peerj.5030.

Gdanetz, K., Benucci, G.M.N., Pol, N.V. and Bonito, G., 2017. CONSTAX: a tool for improved taxonomic resolution of environmental fungal ITS sequences. $B M C$ bioinformatics, 18(1), p.538. https://doi.org/10.1186/s12859-017-1952-x.

Glöckner FO, Yilmaz P, Quast C, Gerken J, Beccati A, Ciuprina A, Bruns G, Yarza P, Peplies J, Westram R, Ludwig W., 2017. 25 years of serving the community with ribosomal RNA gene reference databases and tools. J. Biotechnol. https://doi.org/10.1016/j.jbiotec.2017.06.1198.

Gómez, F., Moreira, D., \& López-García, P., 2010. Neoceratium gen. nov., a new genus for all marine species currently assigned to Ceratium (Dinophyceae). Protist, 161(1), 35-54. https://doi.org/10.1016/j.protis.2009.06.004.

Guillou, L., Bachar, D., Audic, S., Bass, D., Berney, C., Bittner, L., Boutte, C. Burgaud, G., de Vargas, C., Decelle, J., del Campo, J., Dolan, J.R., Dunthorn, M., Edvardsen, B., Holzmann, M., Kooistra, W.H.C.F., Lara, E., Le Bescot, N., Logares, R., Mahé, F., Massana, R., Montresor, M., Morard, R., Not, F., Pawlowski, J., Probert, I., Sauvadet, A.L., Siano, R., Stoeck, T., Vaulot, D., Zimmermann, P., Christen R. 2013. The Protist Ribosomal Reference database $\left(\mathrm{PR}^{2}\right)$ : a catalog of unicellular eukaryote Small Sub-Unit rRNA sequences with curated taxonomy. Nucleic Acids Research. 41:D597-604. https://doi.org/10.1093/nar/gks1160.

Guiry, M.D. \& Guiry, G.M. 2020. AlgaeBase. World-wide electronic publication, National University of Ireland, Galway. https://www.algaebase.org.

Hibbett, D.S., Blackwell, M., James, T.Y., Spatafora, J.W., Taylor, J.W. and Vilgalys, R., 2018. Phylogenetic taxon definitions for Fungi, Dikarya, Ascomycota and Basidiomycota. IMA fungus, 9(2), pp.291-298. https://doi.org/10.5598/imafungus.2018.09.02.05.

Maddison, D. R. and K.-S. Schulz (eds.) 2007. The Tree of Life Web Project. Internet address: http://tolweb.org.

McDonald, D., Price, M.N., Goodrich, J., Nawrocki, E.P., DeSantis, T.Z., Probst, A., Andersen, G.L., Knight, R. and Hugenholtz, P., 2012. An improved Greengenes taxonomy with explicit ranks for ecological and evolutionary analyses of bacteria and archaea. The ISME journal, 6(3), pp.610-618. https://doi.org/10.1038/ismej.2011.139. 
646

647

648

649

650

651

652

653

654

655

656

657

658

659

660

661

662

663

664

665

666

667

668

669

670

671

672

673

674

675

676

677

678

679

680

681

682

683

684

685

686

687

688

689

690

691

McIntyre, A.B., Ounit, R., Afshinnekoo, E., Prill, R.J., Hénaff, E., Alexander, N., Minot, S.S., Danko, D., Foox, J., Ahsanuddin, S., Tighe, S., Hasan, N.A., Subramanian, P., Moffat, K., Levy, S., Lonardi, S., Greenfield, N., Colwell, R.R., Rosen, G.L., Mason, C.E. 2017. Comprehensive benchmarking and ensemble approaches for metagenomic classifiers. Genome biology, 18(1), p.182. https://doi.org/10.1186/s13059-017-1299-7.

Medlin, L., Elwood, H.J., Stickel, S. and Sogin, M.L., 1988. The characterization of enzymatically amplified eukaryotic 16S-like rRNA-coding regions. Gene, 71(2), pp.491499. https://doi.org/10.1016/0378-1119(88)90066-2.

Murali, A., Bhargava, A. and Wright, E.S., 2018. IDTAXA: a novel approach for accurate taxonomic classification of microbiome sequences. Microbiome, 6(1), pp.1-14. https://doi.org/10.1186/s40168-018-0521-5.

H. Pagès, P. Aboyoun, R. Gentleman and S. DebRoy (2019). Biostrings: Efficient manipulation of biological strings. $\mathrm{R}$ package version 2.54.0.

Parada, A.E., Needham, D.M. and Fuhrman, J.A., 2016. Every base matters: assessing small subunit rRNA primers for marine microbiomes with mock communities, time series and global field samples. Environmental Microbiology, 18(5), pp.1403-1414. https://doi.org/10.1111/1462-2920.13023.

R Core Team (2019). R: A language and environment for statistical computing. R Foundation for Statistical Computing, Vienna, Austria. URL https:/www.R-project.org/.

Ratnasingham, S. and Hebert, P.D., 2007. BOLD: The Barcode of Life Data System (http://www.barcodinglife. org). Molecular Ecology Notes, 7(3), pp.355-364. https://doi.org/10.1111/j.1471-8286.2007.01678.x.

Schloss, P.D., Westcott, S.L., Ryabin, T., Hall, J.R., Hartmann, M., Hollister, E.B., Lesniewski, R.A., Oakley, B.B., Parks, D.H., Robinson, C.J. and Sahl, J.W., 2009. Introducing mothur: open-source, platform-independent, community-supported software for describing and comparing microbial communities. Applied and environmental microbiology, 75(23), pp.7537-7541. https://doi.org/10.1128/AEM.01541-09.

Sieber, C.M., Probst, A.J., Sharrar, A., Thomas, B.C., Hess, M., Tringe, S.G. and Banfield, J.F., 2018. Recovery of genomes from metagenomes via a dereplication, aggregation and scoring strategy. Nature microbiology, 3(7), pp.836-843. https://doi.org/10.1038/s41564018-0171-1.

Silén, L. 1972. Fertilization in the Bryozoa, Ophelia, 10:1, 27-34, https://doi.org/10.1080/00785326.1972.10430099.

Sogin, M.L., Morrison, H.G., Huber, J.A., Welch, D.M., Huse, S.M., Neal, P.R., Arrieta, J.M. and Herndl, G.J., 2006. Microbial diversity in the deep sea and the underexplored "rare

PeerJ reviewing PDF | (2021:03:59589:1:0:NEW 3 Jun 2021) 
692

693

694

695

696

697

698

699

700

701

702

703

704

705

706

707

708

709

710

711

712

713

714

715

716

717

718

719

720

721

722

723

724

725

726

727

728

729

730

731

biosphere". Proceedings of the National Academy of Sciences, 103(32), pp.12115-12120. https://doi.org/10.1073/pnas.0605127103.

Quast C, Pruesse E, Yilmaz P, Gerken J, Schweer T, Yarza P, Peplies J, Glöckner FO, 2013, The SILVA ribosomal RNA gene database project: improved data processing and web-based tools. Nucleic Acids Research. 41 (D1): D590-D596.

https://doi.org/10.1093/nar/gks1219.

Varol, M., Blanco, S., Alpaslan, K. and Karakaya, G., 2018. New records and rare taxa for the freshwater algae of Turkey from the Tatar Dam Reservoir (Elazı̆̆ $)$. Turkish Journal of Botany, 42(4), pp.533-542.

Wang, Q, G. M. Garrity, J. M. Tiedje, and J. R. Cole. 2007. Naïve Bayesian Classifier for Rapid Assignment of rRNA Sequences into the New Bacterial Taxonomy. Applied Environmental Microbiology. 73(16):5261-5267, https://doi.org/10.1128/AEM.00062-07.

Wickham, H., Hester, J., Chang, W., 2020. devtools: Tools to Make Developing R Packages Easier. R package version 2.3.1. https://CRAN.R-project.org/package=devtools.

Wickham, H., François, R., Henry, L., Müller, K., 2020. dplyr: A Grammar of Data Manipulation. R package version 0.8.5. https://CRAN.R-project.org/package=dplyr.

Wickham, H., Bryan, J., 2020. usethis: Automate Package and Project Setup. R package version 1.6.1. https://CRAN.R-project.org/package=usethis.

Wickham, H., 2019. stringr: Simple, Consistent Wrappers for Common String Operations. R package version 1.4.0. https://CRAN.R-project.org/package=stringr.

Wright E.S., 2016. Using DECIPHER v2.0 to Analyze Big Biological Sequence Data in R. The $R$ Journal, 8(1), 352-359.

Woese, C.R. and Fox, G.E., 1977. Phylogenetic structure of the prokaryotic domain: the primary kingdoms. Proceedings of the National Academy of Sciences, 74(11), pp.5088-5090. https://doi.org/10.1073/pnas.74.11.5088.

WoRMS Editorial Board (2020). World Register of Marine Species. Available from https://www.marinespecies.org at VLIZ. Accessed 2020-08-29. doi:10.14284/170

Xie, Y, 2020. knitr: A General-Purpose Package for Dynamic Report Generation in R. R package version 1.28. 


\section{Table 1 (on next page)}

Definitions of terms used in the present manuscript and the ensembleTax package. 


\begin{tabular}{|l|l|}
\hline Term & Definition \\
\hline $\begin{array}{l}\text { Taxonomic } \\
\text { nomenclature }\end{array}$ & $\begin{array}{l}\text { A specific framework of naming and ranking conventions } \\
\text { employed by a reference database. }\end{array}$ \\
\hline Taxonomy table & $\begin{array}{l}\text { A collection of ASV sequences (or ASV identifiers) sampled from } \\
\text { the environment with corresponding taxonomic assignments. }\end{array}$ \\
\hline Taxonomy & An organism's taxonomic identity. \\
\hline Taxonomic assignment & The predicted taxonomy for an ASV. \\
\hline $\begin{array}{l}\text { Ensemble taxonomic } \\
\text { assignment }\end{array}$ & $\begin{array}{l}\text { A taxonomic assignment for an ASV determined from several } \\
\text { independent taxonomic assignment methods. Synonymous with } \\
\text { "consensus taxonomic assignment". }\end{array}$ \\
\hline Taxonomic synonyms & \begin{tabular}{l} 
Taxonomic names with equivalent meaning. \\
"Lower" taxonomic ranks would correspond to lower branches in \\
a phylogenetic tree (e.g. species is a lower rank than class). \\
\hline $\begin{array}{l}\text { taxonomigher } \\
\text { axanks }\end{array}$
\end{tabular} \\
\hline
\end{tabular}

1 


\section{Figure 1}

Schematic diagram of potential ensembleTax R package workflows.

The red dashed line separates components of the amplicon sequence data analysis workflow that must be completed prior to use of the ensembleTax package from those that can be addressed with the ensembleTax package. 
Fig 1

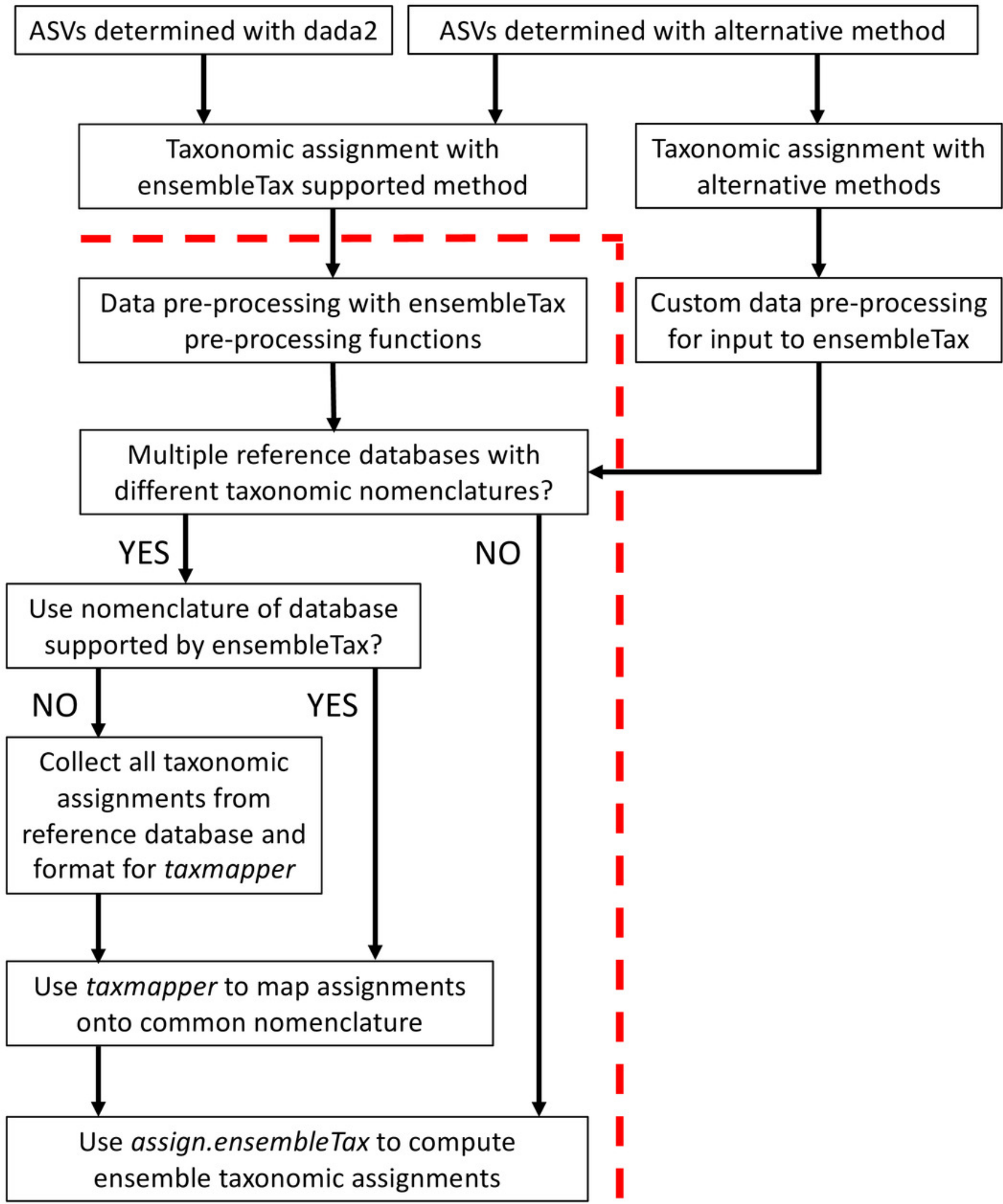




\section{Figure 2}

Schematic diagram illustrating the approach employed by the taxmapper algorithm to map taxonomic assignments onto different taxonomic nomenclatures.

An example input taxonomic assignment and synonymous (through rankD and rank4) entry in a different taxonomic nomenclature are provided as inputs to the taxmapper algorithm. Three examples of mapped taxonomic assignments (A, B, C) are shown based on adjustments to taxmapper parameters indicated by arrows in the flow chart. 
Reference database taxonomic nomenclature:

\begin{tabular}{|c|c|c|c|c|c|}
\hline \multicolumn{2}{|c|}{ RankA } & \multicolumn{2}{|c|}{ RankB } & RankC & RankD \\
\hline \multicolumn{2}{|c|}{ Eukaryota } & Stram & nopila & Bacillariophyta & Pseudonitzschia \\
\hline \multicolumn{6}{|c|}{ Input taxonomy table: } \\
\hline ASV & \multicolumn{2}{|c|}{ Rank1 } & Rank2 & Rank3 & Rank4 \\
\hline ASV1 & \multicolumn{2}{|c|}{ Eukaryota } & Clade_X & Diatomea & Pseudo-nitzschia \\
\hline
\end{tabular}

\section{taxmapper operations:}

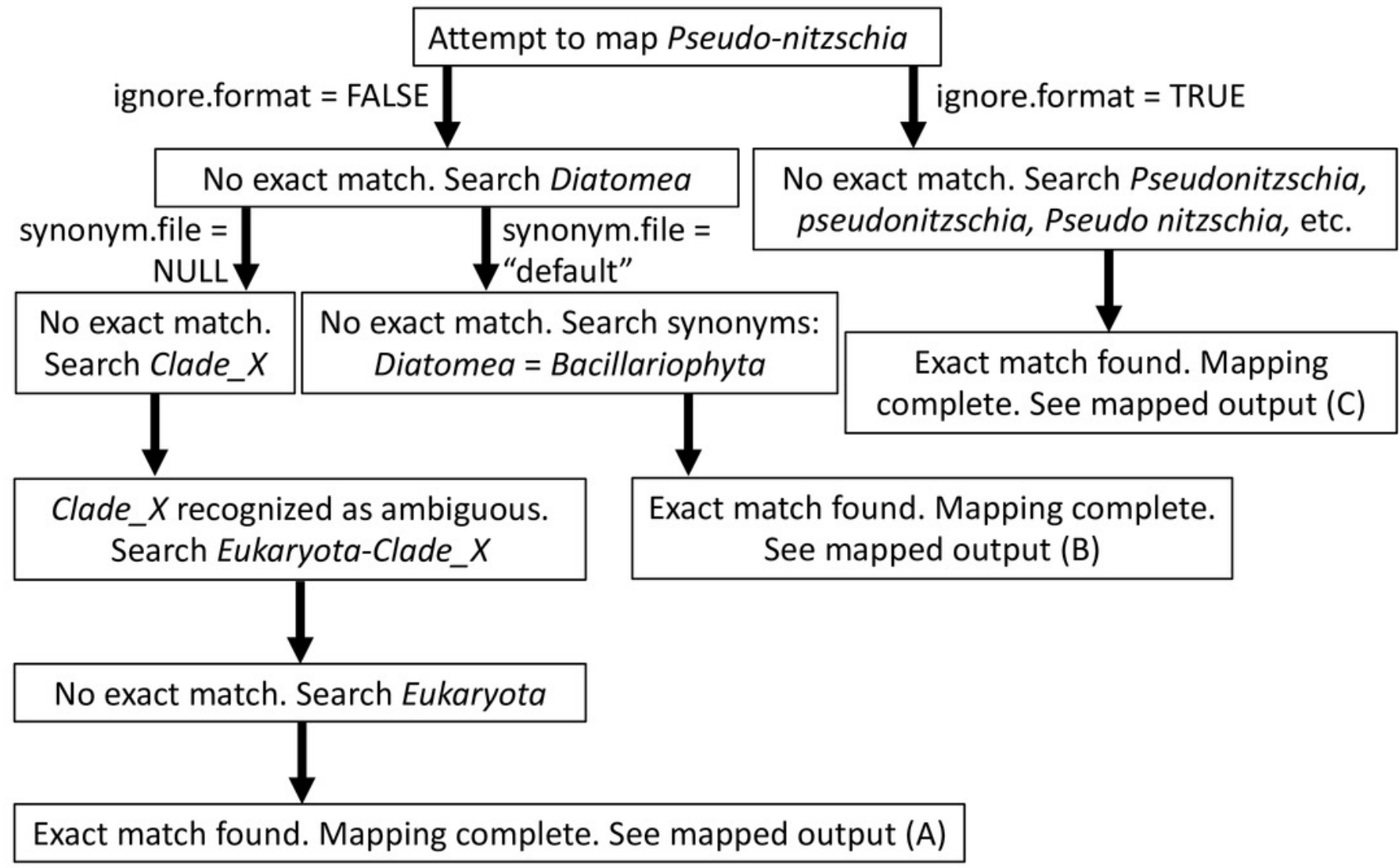

Mapped output taxonomy tables for different parameter spaces:

\begin{tabular}{|l|l|l|l|l|l|l|}
\hline & ASV & RankA & RankB & RankC & RankD & RankE \\
\hline (A) & ASV1 & Eukaryota & NA & NA & NA & NA \\
\hline (B) & ASV1 & Eukaryota & Stramenopila & Bacillariophyta & NA & NA \\
\hline (C) & ASV1 & Eukaryota & Stramenopila & Bacillariophyta & Pseudonitzschia & NA \\
\hline
\end{tabular}




\section{Figure 3}

Schematic diagram demonstrating ensemble taxonomic assignment determinations by the assign.ensembleTax algorithm for different combinations of input taxonomic assignments and arguments.

(A) shows example ensemble taxonomic assignment determinations for a single ASV based on two conflicting methods, illustrating the potential for ensemble methods to remove annotation errors introduced by individual taxonomic assignment methods and/or for users to select which taxonomic assignment methods are prioritized in the event of disagreements between individual methods. (B) shows example ensemble taxonomic assignment determinations for a single ASV based on three individual methods that vary in both the number of ranks with assigned names and the identity of names where they are assigned.

Input taxonomic assignments:

(A) Ensemble determinations for two methods with conflicting taxonomy predictions

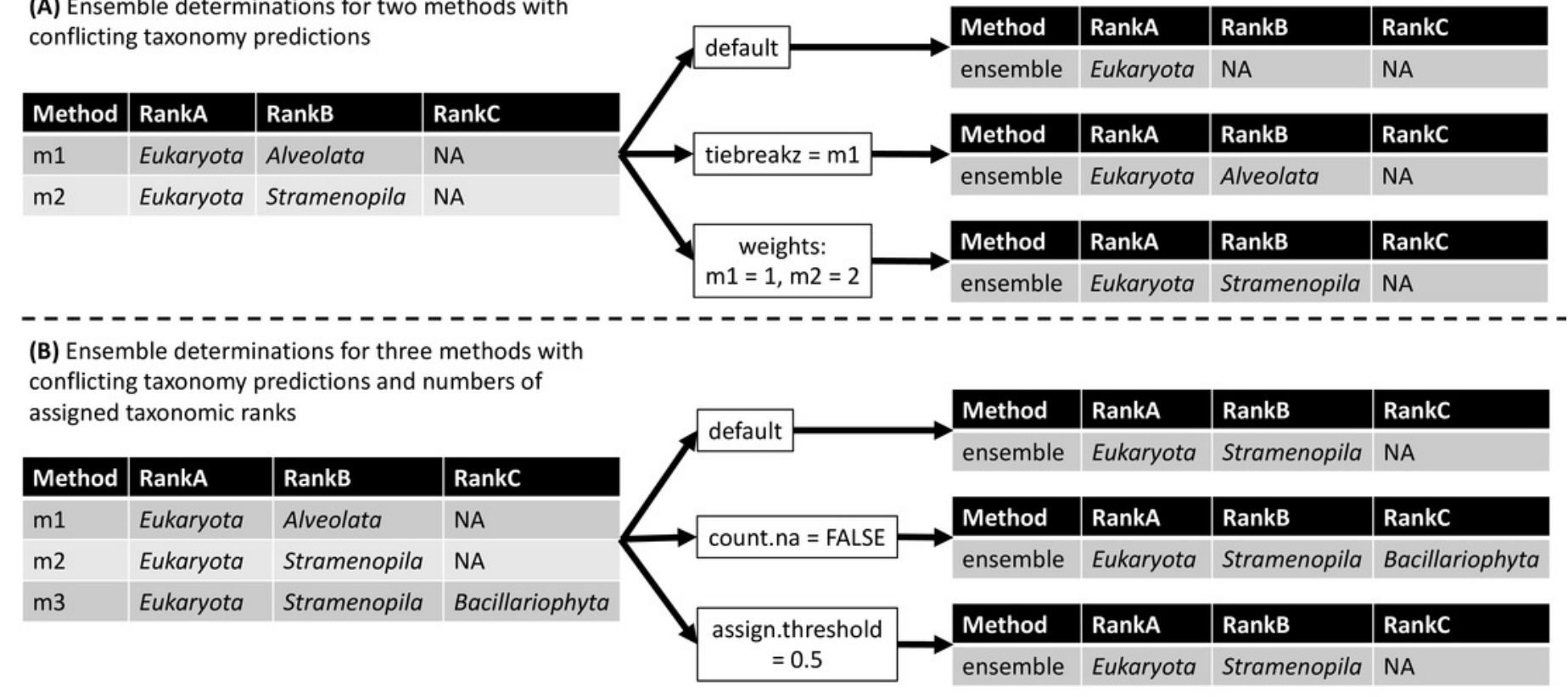

assign.ensembleTax parameters:

Ensemble taxonomic assignments: 


\section{Figure 4}

Comparisons of taxonomic assignments predicted by individual methods with ensemble taxonomic assignments for a large data set of protistan ASVs sampled from the coastal ocean.

(A, B) show rank-wise comparisons of the proportion of ASVs that remained unassigned (bootstrap values $<60 \%$ in bayes-pr2 and bayes-silva, $<50 \%$ in idtax-pr2 and idtax-silva) in each taxonomy table using (A) the Prostistan Ribosomal Reference Database v4.12.0 (pr2) and (B) the SILVA SSU $\mathrm{nr}$ reference database v138 (silva) taxonomic nomenclatures. (C, D, E, F) compare the taxonomic assignments predicted by individual (bayes-pr2, bayes-silva, idtaxpr2, idtax-silva) and ensemble (ensemble1, ensemble2) methods with taxonomic assignments predicted by ensemble methods favoring (C, D) more robust taxonomic assignments supported by multiple methods or $(E, F)$ assigning taxonomy to more ranks for a higher proportion of ASVs using the (C, E) pr2 or (D, F) silva taxonomic nomenclatures. In (C, $D, E, F)$, "Agree" indicates that the two taxonomic assignment methods agree in both the number of ranks assigned and the identity of assigned taxonomic names. "More/less ranks assigned" indicates that where taxonomic names are assigned they agree across the two methods, but more or fewer clade names were predicted by one method than the other. 
Taxonomy table $\square$ idtax-pr2 $\square$ idtax-silva $\square$ ensemble1

(A)

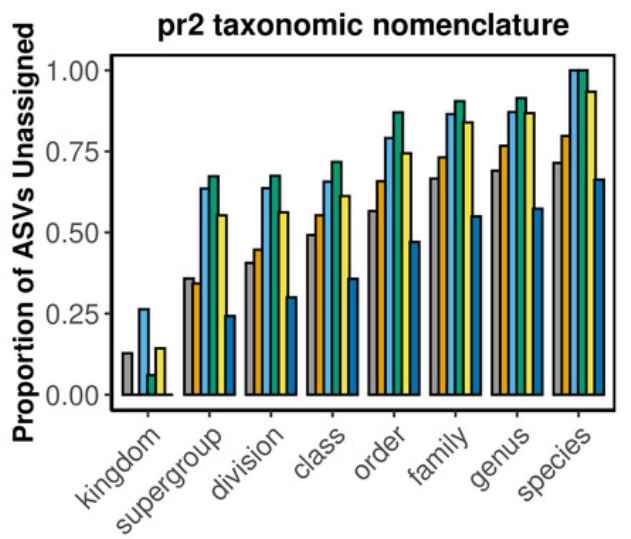

(C)

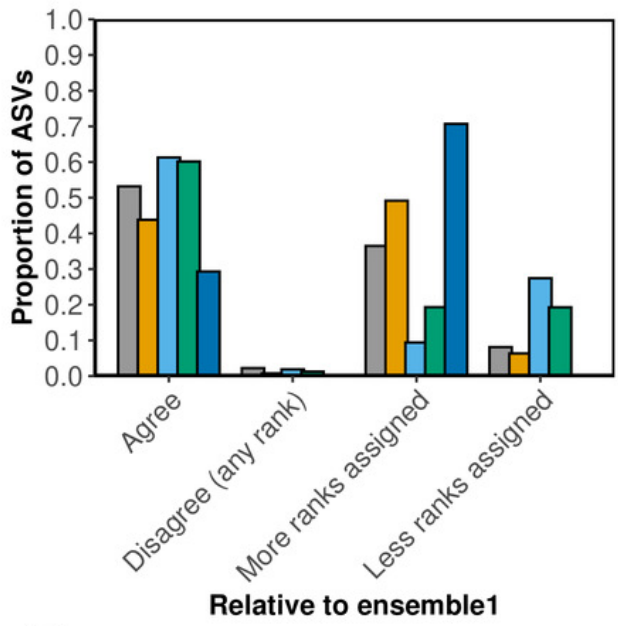

(E)

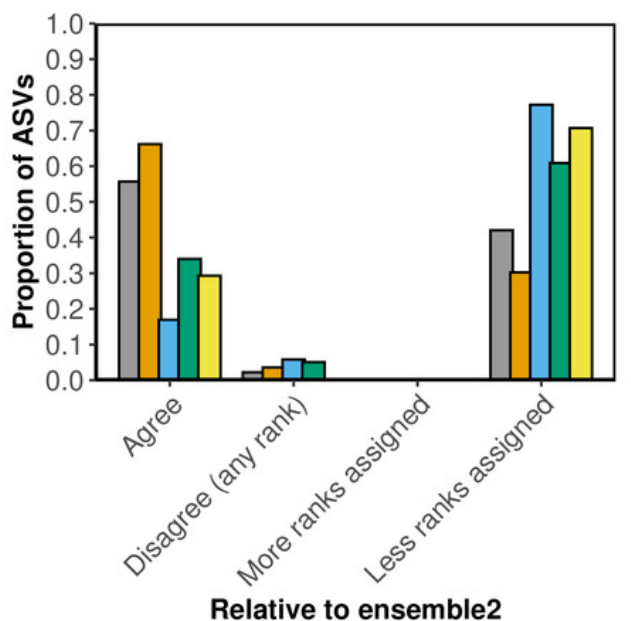

(B)

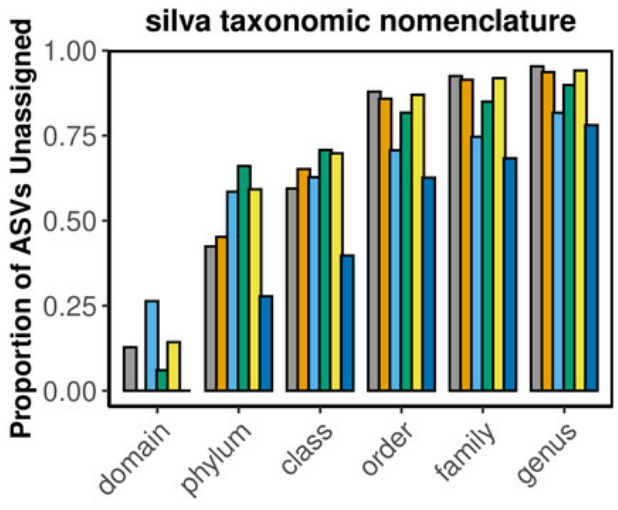

(D)

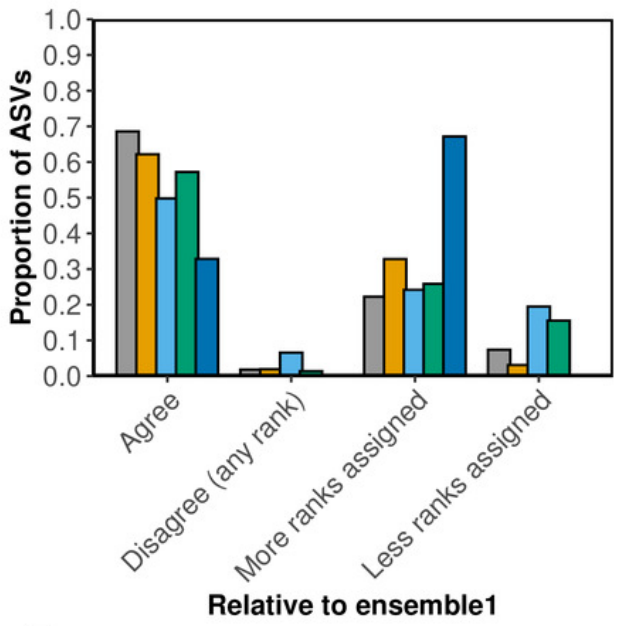

(F)

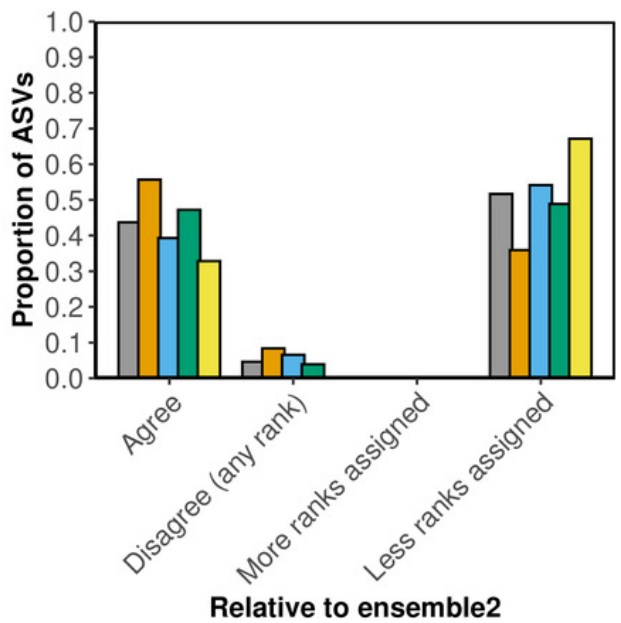

